\title{
Operant behavior in transition: FI to FI
}

\author{
FRANK A. LOGAN \\ University of New Mexico, Albuquerque, New Mexico 87131
}

\begin{abstract}
Three rats received extensive initial training on an FI 2-min schedule of reinforcement and were then shifted to an FI 30-sec schedule. The length of the postreinforcement pause very rapidly decreased, suggesting that the rats were "surprised" by the changed schedule. The subsequent shift back to the FI 2-min schedule led to a gradual change in performance, with responding appropriate to both intervals occurring.
\end{abstract}

The great majority of studies in the experimental analysis of operant behavior have concerned the steady state performance resulting from prolonged exposure to one or another schedule of reinforcement. This approach was exemplified in Behavior of Organisms (Skinner, 1938) and epitomized in Schedules of Reinforcement (Ferster \& Skinner, 1957). To date, a very large number of schedules have been subjected to such an analysis.

There are at least two reasons why considerable interest should also be directed toward the dynamic processes involved when behavior is in transition as a result of a change in the prevailing schedule of reinforcement. One reason is simply that a change in behavior is typically listed as one of the defining features of learning. Hence, insofar as one is interested in learning, per se, an analysis of behavior in transition is necessarily implicated.

The second reason for an interest in behavior following a change in the schedule of reinforcement relates to the classic question of what is learned. As cogently argued by Campbell (1954), original performance is often subject to several interpretations that can, to some extent at least, be evaluated by what he called the transposition procedure. The basic idea is that an organisms's response to a change in the contingencies can often be predicted by hypotheses about what was learned from the earlier experiences.

There are undoubtedly several reasons why little attention has been paid to operant behavior in transition, but the most obvious is the sheer number of logically possible changes in the schedule of reinforcement. Especially when one includes several parametric values for each schedule, the number of original schedules is already very large and the number of transitions is that large number squared. Furthermore, if one is willing to consider a second change, which may prove to be even more informative than the first, the number of possible

This research was supported in part by the National Science Foundation and was reported at the meeting of the Psychonomic Society, November 1978, San Antonio, Texas. Requests for reprints may be addressed to the author, Department of Psychology, University of New Mexico, Albuquerque, New Mexico 87131. sequences of schedules is the cube of the large number of original schedules. Especially if one were to adopt a purely empirical approach to the topic, one would quickly be overwhelmed by the enormity of the task.

Nevertheless, a few studies have been done that involve a sustained change in the schedule of reinforcement following extensive exposure to some other schedule. (I am excluding studies of mixed and multiple schedules, which are, themselves, typically analyzed after a large number of shifts back and forth between the schedules.) Illustrative is the work by Weiss (1970), who demonstrated a computer analysis of changing interresponse time distributions when various differential reinforcement schedules were imposed.

The present research was conceived as a preliminary study using two FI schedules that would generate distinctive terminal performance patterns, so that intermediate forms of behavior in transition might be readily discernible.

\section{METHOD}

\section{Subjects}

Three male hooded rats, 90 days old at the start of the experiment, were obtained from the animal colony in the Department of Psychology at the University of New Mexico. They were maintained at $85 \%-90 \%$ ad-lib body weight by restricted daily feedings, with water freely available in their individual home cages.

\section{Apparatus}

Three Lehigh Valley operant conditioning chambers, programmed for delivery of $20-\mathrm{mg}$ Noyes reinforcement pellets, were employed. Programming and recording were done with a combination of solid state and electromechanical equipment located in the adjacent room; exhaust fans in each chamber helped mask any sounds from the equipment. Data were recorded on magnetic tape for later analysis.

\section{Procedure}

The rats were autoshaped by concurrently programming an FI 2-min +30 -sec limited-hold schedule with free food delivered on an FT 2.5-min schedule. That is to say, if the rat pressed the bar during a $30-\mathrm{sec}$ period starting 2 min after the preceding reinforcer, reinforcement was immediate and the next FI started. If the rat did not press the bar during the 30 -sec limited hold, a reinforcer was automatically delivered and the next FI started. With this procedure, the rats never experienced reinforcers occurring less than 2 min apart. As soon as a rat was obtaining a 
substantial number of pellets by barpressing, the free food delivery and the limited-hold features of the schedule were dropped, leaving the rat on an FI 2-min schedule of reinforcement. (One additional rat failed to autoshape within 10 days using this procedure and was dropped from the experiment.)

The rats were run daily for a 50 -min session for 90 days on the FI 2-min schedule, which was in effect for the first $10 \mathrm{~min}$ of Day 91 before the schedule was changed to FI $30 \mathrm{sec}$. There were no explicit stimuli associated with this change in the schedule of reinforcement. Then, after 30 days on the FI $30-\mathrm{sec}$ schedule, the reverse change back to the FI 2-min schedule was effected for the final 8 days of the experiment.

\section{RESULTS}

The rats' behavior following the first change in reinforcement schedule is best depicted in terms of the latency of the first response after each reinforcement (the "postreinforcement pause"). These data are plotted in Figure 1, which shows the three rats individually, as well as the mean latency. On the five trials preceding the shift, there was considerable variability, but the average latency ran well over $1 \mathrm{~min}$, indicating reasonably good adjustment to the FI 2-min schedule. On the very first trial after encountering the changed schedule, all rats showed a substantial decrease in latency, and within five trials, the average latency was down to about $20 \mathrm{sec}$. This decrease in latency continued even though the new schedule provided reinforcement no sooner than $30 \mathrm{sec}$ after each preceding reinforcer. (The data following the first response in each interval are not depicted graphically because they can be described adequately as a steady burst of responses until reinforcement was received.)

The effect of the change back to the initial FI 2-min schedule is depicted in Figures 2 and 3. This phase of the study lasted only 8 days, but this was sufficient to

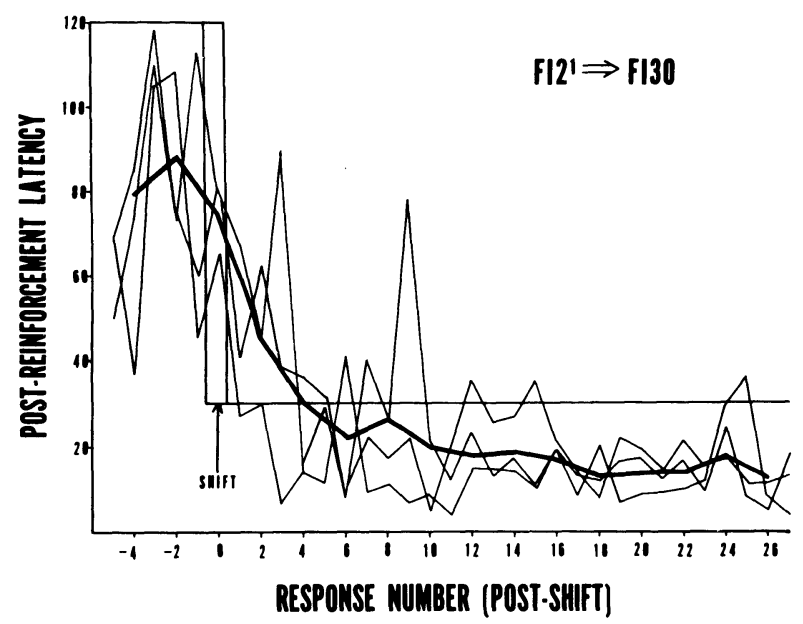

Figure 1. Postreinforcement latency (pause) after the last five reinforcements before the change from an FI 2-min schedule to an FI 30-sec schedule, and subsequent latencies. The narrow curves show the individual latencies and the heavy curve is a sliding average of the three rats over three reinforcers.

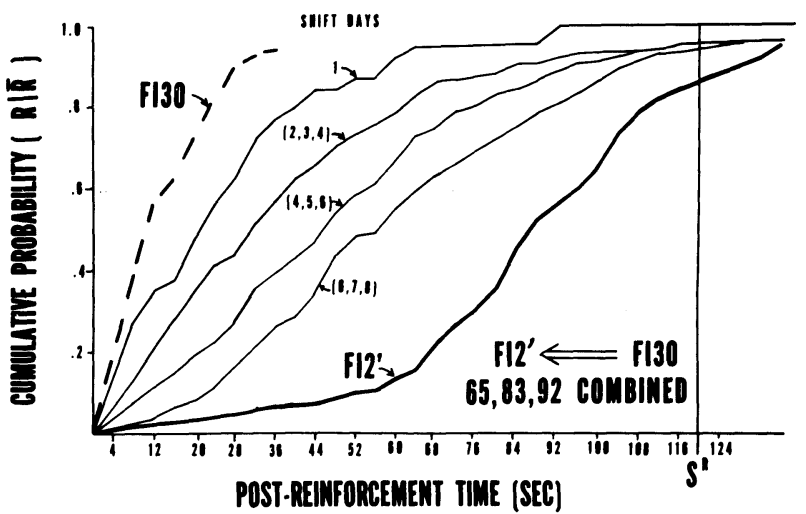

Figure 2. Cumulative probability of the first response occurring during successive 8 -sec time bins during the FI. Depicted are the averages over blocks of days after the change back to an FI 2-min schedule after 30 days' exposure to an FI 30-sec schedule. Terminal performance on the two schedules is depicted by the heavy curves.

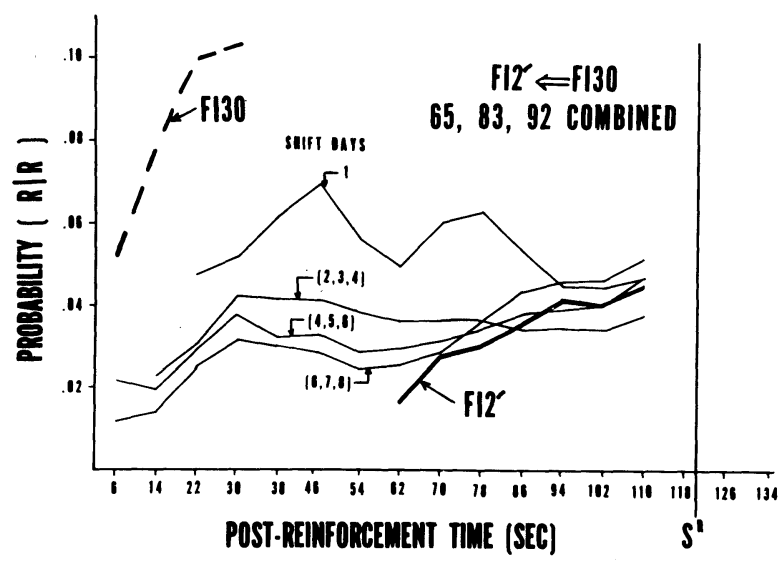

Figure 3. Momentary probability of responding during successive 8 -sec time bins during the FI, conditional on the first response having been emitted before that time. Depicted are the averages over blocks of days after the change back to an FI 2-min schedule after 30 days' exposure to an FI 30-sec schedule. Terminal performance on the two schedules is depicted by the heavy curves.

observe the essential outcome. In this case, there was a gradual shift back toward the performance appropriate to the FI 2-min schedule. Noteworthy in Figure 3 is a tendency to emit responses at about the time of reinforcement on the FI 30-sec schedule.

\section{DISCUSSION}

Descriptively, the behavior following the first change in the schedule of reinforcement (from FI 2 min to FI $30 \mathrm{sec}$ ) could be considered to be an operant analogue of the successive positive contrast effect sometimes found in instrumental (discretetrial) procedures. That phenomenon, initially called the "elation effect" (Crespi, 1942), is that rats shifted from a small to a large reward in a straight alley show a rapid increase in running speed, 
temporarily exceeding the speed normally resulting from the large reward. In like fashion, the present animals very quickly shifted from the long postreinforcement pauses appropriate for the long FI schedule to pauses even shorter than appropriate for the short FI schedule.

However, this observed change in behavior may have greater conceptual significance than simply treating it as another instance of contrast effects. Consider an informal, cognitive analysis of behavior under an FI schedule of reinforcement. The subject is viewed as judging time according to some fallible internal clock, waiting until it judges the requisite time to have passed, and responding when it expects reinforcement for doing so. According to such an analysis, there is no reason for there to be a rapid change in behavior when the schedule is changed. The rat presses the bar when it expects reinforcement, it indeed receives reinforcement, and hence there is nothing unexpected about the event. The fact that the rats do change their behavior abruptly, beginning immediately with the first reinforcement encountered after the change in the schedule, quite convincingly rules out any analysis based on such reasoning.

It is important to note that the rats had never before experienced an interreinforcement interval of less than $2 \mathrm{~min}$, and hence the rapid change in behavior cannot be viewed as a return to an earlier rate of responding. Nevertheless, the rats were obviously "surprised" by the first reinforcer after the change in the schedule; they clearly did not "expect" to be reinforced immediately upon beginning to emit premature responses. This suggests that such early responses to an FI schedule are controlled less by explicit response-reinforcer contingencies than by implicit stimulus-reinforcer contingencies, provided one accepts the presence of some discriminable stimulus features related to elapsed time since the preceding reinforcer. According to this view, premature responses on an FI schedule represent adjunctive rather than instrumental behavior.

My theoretical account in terms of hybrid theory (Logan, 1979 ) is that premature responses begin to occur when, as the stimulus trace of the preceding reinforcer decays, the net excess of positive expectancy over negative expectancy exceeds a randomly variable threshold, which means simply that no other behavior has a higher response tendency. An FI schedule thus induces an organism to emit premature responses, and reinforcement becomes associated, not with a single response, but with a chain of responses correlated with a decaying stimulus memory trace of the preceding reinforcer. Therefore, the occurrence of reinforcement after the first response is indeed an unusual stimulus event leading to disinhibition as well as counterconditioning of the negative expectancies.

The results of the second change in schedule can be understood in these same terms. Specifically, in this case, adjustment to the change in schedule requires the reacquisition of a negative expectancy (i.e., extinction) associated with the short-lived elements of the stimulus trace of the preceding reinforcer. Since the first change did not involve counterconditioning of the positive expectancy associated with the long-lived elements, appropriate behavior should reappear when these hypothetical stimulus events again occur. Hence, there is a return to a long-FI scallop, sometimes preceded by responses at the short-FI time.

\section{REFERENCES}

Campbell, D. T. What is learned via the transposition experiment. Psychological Review, 1954, 61, 161-174.

Crespi, L. P. Quantitative variation in amount of incentive. American Journal of Psychology, 1942, 55, 457-517.

Ferster, C. B., \& Skinner, B. F. Schedules of reinforcement. New York: Appleton-Century-Crofts, 1957.

LogAn, F. A. Hybrid theory of operant conditioning. Psychological Review, 1979, 86, 507-541.

Skinner, B. F. Behavior of organisms. New York: AppletonCentury, 1938.

WEiss, B. The fine structure of operant behavior during transition states. In W. N. Schoenfeld (Ed.), Theory of reinforcement schedules. New York: Appleton-Century-Crofts, 1970.

(Received for publication October 5, 1981.) 